\title{
Short-term variation in the galactic cosmic ray intensity measured with the PAMELA experiment.
}

Riccardo Munini*1, Valeria di Felice $^{2,3}$, M. Boezio ${ }^{1}$, O. Adriani ${ }^{4,5}$, G. C. Barbarino ${ }^{6,7}$, G. A. Bazilevskaya ${ }^{8}$, R. Bellotti ${ }^{9,10}$, E. A. Bogomolov ${ }^{11}$, M. Bongi ${ }^{3,5}$, V. Bonvicini ${ }^{1}$, S. Bottai ${ }^{5}$, A. Bruno ${ }^{9,10}$, F. Cafagna ${ }^{10}$, D. Campana ${ }^{7}$,P. Carlson ${ }^{12}$, M. Casolino ${ }^{2,14}$, G. Castellini ${ }^{15}$, C. De Donato ${ }^{2}$, C. De Santis ${ }^{13}$, A. M. Galper ${ }^{16}$, A. V. Karelin ${ }^{16}$, S. V. Koldashov ${ }^{16}$, S. Koldobskiy ${ }^{16}$, S. Y. Krutkov ${ }^{11}$, A. N. Kvashnin ${ }^{8}$, A. Leonov ${ }^{16}$,V. Malakhov ${ }^{16}$, L. Marcelli ${ }^{13}$, M. Martucci ${ }^{13,17}$, A. G. Mayorov ${ }^{16}$, W. Menn ${ }^{18}$, M. Merge ${ }^{2,13}$, V. V. Mikhailov ${ }^{16}$,E. Mocchiutti ${ }^{1}$, A. Monaco ${ }^{9,10}$, N. Mori ${ }^{5}$, G. Osteria ${ }^{7}$, B. Panico ${ }^{7}$ P. Papini ${ }^{5}$, M. Pearce ${ }^{12}$, P. Picozza ${ }^{2,13}$, M. Ricci ${ }^{17}$, S. B. Ricciarini ${ }^{15}$, M. Simon ${ }^{18}$, R. Sparvoli ${ }^{2,13}$, P. Spillantini ${ }^{4,5}$, Y. I. Stozhkov ${ }^{8}$, A. Vacchi ${ }^{1,19}$, E. Vannuccini ${ }^{5}$, G. Vasilyev ${ }^{11}$, S. A. Voronov ${ }^{16}$, Y. T. Yurkin ${ }^{16}$, G. Zampa ${ }^{1}$, N. Zampa ${ }^{1}$, M. S. Potgieter ${ }^{20}$, E. C. Christian ${ }^{21}$, G. A. de Nolfo ${ }^{21}$, I. Richardson ${ }^{21,22}$, J. M. Ryan ${ }^{23}$, S. Stochaj ${ }^{24}$,

${ }^{1}$ INFN, Sezione di Trieste I-34149 Trieste, Italy

2 INFN, Sezione di Rome "Tor Vergata”, I-00133 Rome, Italy

${ }^{3}$ Agenzia Spaziale Italiana (ASI) Science Data Center, I-00044 Frascati, Italy

${ }^{4}$ University of Florence, Department of Physics, I-50019 Sesto Fiorentino, Florence, Italy

5 INFN, Sezione di Florence, I-50019 Sesto Fiorentino, Florence, Italy

${ }^{6}$ University of Naples "Federico II", Department of Physics, I-80126 Naples, Italy

${ }^{7}$ INFN, Sezione di Naples, I-80126 Naples, Italy

${ }^{8}$ Lebedev Physical Institute, RU-119991, Moscow, Russia

${ }^{9}$ University of Bari, Department of Physics, I-70126 Bari, Italy

${ }^{10}$ INFN, Sezione di Bari, I-70126 Bari, Italy

${ }^{11}$ Ioffe Physical Technical Institute, RU-194021 St. Petersburg, Russia

${ }^{12}$ KTH, Department of Physics, and the Oskar Klein Centre for Cosmoparticle Physics, AlbaNova University Centre, SE-10691 Stockholm, Sweden

13 University of Rome "Tor Vergata”, Department of Physics, I-00133 Rome, Italy

${ }^{14}$ RIKEN, Advanced Science Institute, Wako-shi, Saitama, Japan

15 IFAC, I-50019 Sesto Fiorentino, Florence, Italy

${ }^{16}$ National Research Nuclear University MEPhI, RU-115409 Moscow

${ }^{17}$ INFN, Laboratori Nazionali di Frascati, Via Enrico Fermi 40, I-00044 Frascati, Italy

18 Universität Siegen, Department of Physics, D-57068 Siegen, Germany

${ }^{19}$ University of Udine, Department of Mathematics and Informatics, I-33100 Udine, Italy

${ }^{20}$ North-West University, Centre for Space Research,2520 Potchefstroom, South Africa

${ }^{21}$ Heliophysics Division, NASA Goddard Space Flight Ctr, Greenbelt, MD, USA.

${ }^{22}$ GPHI and Department of Astronomy, University of Maryland, College Park

23 Space Science Center, University of New Hampshire, Durham, NH, USA.

${ }^{24}$ Electrical and Computer Engineering, New Mexico State University, Las Cruces, NM, USA. 
New results on the galactic cosmic ray (GCR) short-term intensity variation associated with Forbush decrease and co-rotating interaction regions (CIRs) measured by the PAMELA instrument between November 2006 and March 2007 are presented. Most of the past measurements on Forbush decrease events were carried out with neutron monitor detector. This tecnique allows only indirect detection of the overall GCR intensity over an integrated energy range. For the first time, thanks to the unique features of the PAMELA magnetic spectrometer, the Forbush decrease associated with the December $13^{\text {th }}$ coronal mass ejection (CME) was studied in a wide rigidity range $(0.4-20 \mathrm{GV})$ and for different species of GCRs detected directly in space. Using GCR protons, the amplitude and the recovery time of the Forbush decrease were studied for ten rigidity interval with a temporal resolution of one day. For comparison the helium and the electron intensity over time were also studied. The temporal evolution of the helium and proton intensity was found in good agreement while the electrons show, on average, a faster recovery time. This was interpreted as a charge-sign dependence introduced by drift motion experienced by the low rigidity $(<5 \mathrm{GV})$ GCRs during their propagation through the heliosphere. Moreover a clear 13.5 days cyclical variation was observed in the GCR proton intensity after the Forbush decrease. This phenomena could be interpreted as an effect of prominent structures of compressed plasma in the solar wind, i.e. CIRs, or to the latitudinal gradient due to the crossing of the heliospheric current sheet (HCS).

35th International Cosmic Ray Conference - ICRC2017

10-20 July, 2017

Bexco, Busan, Korea

*Speaker. 


\section{Introduction}

The solar environment heavily affects CR measurements performed at Earth below $\sim 30 \mathrm{GV}$. Before reaching the Earth, GCRs propagate through the heliosphere, the region of space formed by the continuous outflow of plasma from the Sun surface also called solar wind (SW). In addition, the magnetic field of the Sun freezes into the solar wind plasma and propagates through the heliosphere. This originate the so called heliospheric magnetic field (HMF) [1]. The charged particles of galactic origin, traveling through the interplanetary medium, interacts with the SW and the HMF. As a consequence their spectra are modified in intensity and shape with respect to the local interstellar spectrum (LIS) [2].In addition, in response to the 11 years solar cycle, a long-term modulation of the GCRs is observed. The solar modulation of GCR is anti-correlated with respect to the solar cycle since the particle fluxes reach their maximal intensity during periods of low solar activity.

On top of the long-term solar modulation, short-term modulation effects also occur. For example, the near Earth GCR intensity is greatly modified by transient phenomena in the solar environment. In particular, interplanetary transients such as coronal mass ejection (CME) can induce a Forbush decrease, i.e. a sudden suppression of GCR intensity near the Earth [3]. Such a phenomena can last up to several days and suppress the GCR intensity measured during quite Sun condition of about $30 \%-40 \%$.

In addition, recurrent short-term GCR decreases have been measured in association with the passage of CIRs. Such regions of compressed plasma, formed at the leading edges of high-speed solar wind streams originating from coronal holes and interacting with the preceding slow solar wind, are a well known cause of periodic CR decreases [4],[5]. The periodicity of the CIR-associated GCR intensity variation it's about 27 days (Carrington rotation) or less, depending on the coronal holes number and position over the Sun surface. Many instrument already studied this periodic temporal variation (e.g. Ulysses ecc [6]). As an example, [7] studied GCR during the recent solar $A<0^{1}$ solar minimum, showing recurrent cosmic ray decreases with a clear 27 day solar-rotation modulation.

In this work we studied short-term GCR variations during the extraordinary deep and long solar minimum between solar cycles 23 and 24 [8] as observed by the PAMELA space mission. In particularly we analyzed variations occurred from November 2006 to March 2007. Solar minimum periods are particularly suited to reveal transient phenomena like Forbush decrease and CR suppression associated to CIRs. Remarkably, this period was characterized by very stable heliospheric conditions, except for the powerful solar events occurred on the December 13th/14th 2006. In particular, the first perturbation happened on December 13, when a X3.4/4B solar flare occurred in the active region NOAA 10930, producing a full-halo CME whose forward shock reached Earth at 1438 UT on December 14. It was then followed by the S06W46 X1.5 flare on December 14. The event was unusually powerful for a period of solar minimum and produced a growth of particles below a few $\mathrm{GeV}$ that was recorded by PAMELA and various satellites[9]. Moreover, the passage of the CME caused a Forbush decrease that lasted for several days. Thanks to its quasi-polar orbit the PAMELA instrument has measured the effect of this transient in the GCR intensity in the rigidity range from $400 \mathrm{MV}$ to $20 \mathrm{GV}$. The rigidity reconstruction accuracy and the high collected

\footnotetext{
${ }^{1}$ In the complex sun magnetic field the dipole term nearly always dominates the magnetic field of the solar wind. A is defined as the projection of this dipole on the solar rotation axis.
} 


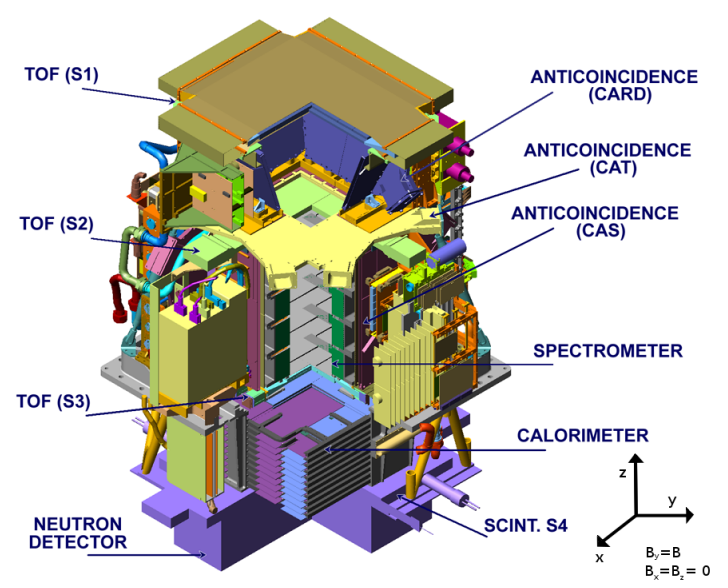

Figure 1: PAMELA and its sub-detectors.

statistics allowed to perform a rigidity dependent study of the Forbush decrease. This extends and completes studies based on other measurements, typically performed on ground, either by neutron monitors or muon telescopes [10]. The performance of these ground based detector are limited since they can only determined an integral flux above an energy threshold which depends on the latitudinal geomagnetic cutoff at which the monitors are placed on the Earth's surface. In addition, the PAMELA instrument allowed to study the GCR temporal evolution comparatively for several particle species like protons, helium and electrons.

The PAMELA instrument also measured a periodic intensity variation with a period of about 13.5 which last for about two month after the December 2006 Forbush decrease. This effect could be associated to the passage of CIRs.

In the following we give details of the data analysis methods used to measure proton, Helium and electron intensities at various rigidities and their evolution in time.

\section{The PAMELA instrument}

PAMELA, Payload for Matter-Antimatter Exploration and Light Nuclei Astrophysics, is a satellite-borne experiment designed to make long duration measurements of the cosmic radiation [11]. Since its launch on June 2006, the instrument has been measuring cosmic ray particles and collecting data for almost 10 years in elliptical orbit, until September 2010, at an altitude ranging between 350 and $610 \mathrm{~km}$ with an inclination of 70 degrees.

The apparatus is schematically shown in Figure 1. The core of the instrument is the magnetic spectrometer, a silicon tracking system in the $0.43 \mathrm{~T}$ magnetic field generated by a permanent magnet. The $300 \mu \mathrm{m}$ thick double-sided Si sensors of the tracking system measure two independent impact coordinates (bending X-view and non-bending Y-view) on each plane, accurately reconstructing the particle deflection, measuring its rigidity (momentum divided by charge) with a maximum detectable rigidity of $1.2 \mathrm{TV}$, and the sign of the electric charge. The instrument geometric factor, as defined by the magnetic cavity, is $21.5 \mathrm{~cm}^{2} \mathrm{sr}$. A system of six layers of plastic scintillators, arranged in three double planes (S1, S2 and S3), provides a fast signal for triggering the data acquisition. Moreover it contributes to particle identification measuring the ionization energy loss, 
and the Time of Flight ( $\mathrm{ToF}$ ) of traversing particles with a resolution of $300 \mathrm{ps,} \mathrm{assuring} \mathrm{charge} \mathrm{par-}$ ticle absolute value determination and albedo particle rejection. The final hadron-lepton discrimination is provided by an electromagnetic imaging W/Si calorimeter, 16.3 radiation lengths and 0.6 interaction lengths deep. Thanks to its longitudinal and transverse segmentation, the calorimeter exploits the different development of electromagnetic and hadronic showers, allowing a rejection power of interacting and non-interacting hadrons at the order of $10^{5}$. A neutron counter contributes to discrimination power by detecting the increased neutron production in the calorimeter associated with hadronic showers compared to electromagnetic ones, while a plastic scintillator, placed beneath the calorimeter, increases the identification of high-energy electrons. The whole instrument is surrounded by an anticoindence system (AC) of three scintillators (CARD, CAS and CAT) for the rejection of background events.

\section{Data analysis}

A set of cuts was developed in order to select a clean sample of protons, helium and electrons from data collected by the PAMELA instrument between November 2006 and March 2007. Only events with a single reconstructed track were selected. The track was required to be located inside a fiducial volume bounded $0.15 \mathrm{~cm}$ from the magnet cavity walls in order to avoid interaction with the magnetic walls which could degrade the tracker performance. Protons and helium nuclei were selected by means of the ionization energy losses in the tracker and ToF planes. In Figure 2 the average ionization energy loss in terms of MIP ${ }^{2}$ inside the tracker planes is shown. Data refers to those collected in flight by the PAMELA instrument between June and December 2006. The black lines represent a constant efficiency selections on the proton (lower bands) and helium (upper bands) nuclei. No isotopic separation (proton/deuterium or ${ }^{3} \mathrm{He} /{ }^{4} \mathrm{He}$ ) were done. Electrons were selected exploiting the PAMELA electromagnetic calorimeter. Several selections based on the topological development of the electromagnetic shower were defined. These selections allowed an almost complete rejection of the, antiproton and pion $^{3}$ contamination in the rigidity range considered. Galactic particles were selected requiring a rigidity value grater than 1.3 times the value of the Stöermer vertical cutoff.

The proton, helium and electron fluxes were finally measured dividing the number of selected events for the selection efficiencies, the live-time and the geometrical factor. To avoid any biases which could introduce systematic temporal variation in the final fluxes, the temporal evolution of the selection efficiencies was studied. The selection efficiencies for the ionization energy losses were found to be constant during the whole time interval under analysis. Also the calorimeter selections were found constant over time. On the contrary the tracker selection efficiency was found to decrease with time. This effect could be due to the random failure of some read-out chips of the silicon mictrostrip detectors. The tracker efficiency was evaluated with two independent procedure.

\footnotetext{
${ }^{2}$ Energy loss is expressed in terms of MIP that is the energy released by a particle which mean energy loss rate in matter is minimum.

${ }^{3}$ Both positive and negative pions are produced by the interaction of primary cosmic rays nuclei with the aluminum container that contains the PAMELA instrument.
} 


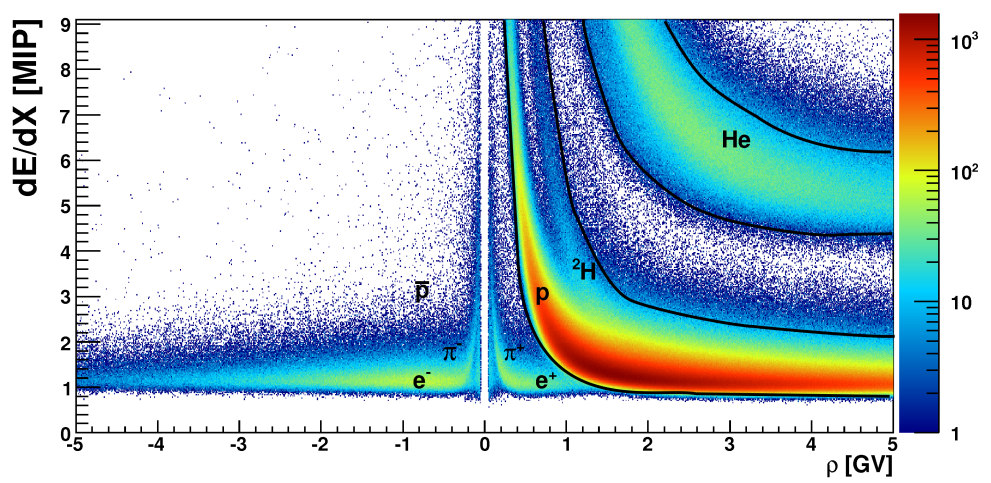

Figure 2: The average ionization losses on the tracker planes as a function of the rigidity measured by the magnetic spectrometer. The heliums, protons, electrons and pions distribution are well separated thanks to the excellent MIP resolution. The upper and the lower bands (black lines) represent the selections for the helium nuclei and proton respectively.

- The PAMELA simulation software (based on GEANT4) was used to generate an isotropical set of proton in the energy range under analysis. The events reconstructed inside the instrumental acceptance were used to measured the energy dependence of the tracker efficiency. The simulation toolkit reproduce the flight configuration of the tracker planes and its temporal evolution. The simulated efficiencies were then evaluated with a temporal resolution of one month.

- A set of non interacting ${ }^{4}$ protons were selected with the calorimeter from flight data. These events were used to measured the tracker selection efficiency. This procedure allows to estimate the efficiency only within an integrated energy range with a lower threshold of few $\mathrm{GeV}$. The high statistics allowed to estimate the weekly integrated efficiency during the period of time under analysis.

The results are shown in Figure 3 (top panel) where both the simulated (red points) and the flight (light green points) tracker efficiency as a function of time are shown. As can be noticed from the bottom panel of Figure 3 an agreement of the order of $2-3 \%$ was found between the simulated and the fligth efficiencies over the whole time interval. Due to the good agreement between the simulated and flight efficiencies, in order to minimize the fluctuation, the final fluxes were calculated using the interpolated values of the simulated efficiencies (red line in Figure 3). The high statistics allowed to measured the proton and helium flux on a daily average while the limited electrons statistics set the smallest time interval in two days.

In order to study the intensity variation each daily flux was normalize to the GCR intensity measured the month before the Forbush decrease, i.e. November 2006. It was then assumed that during the event the solar modulation had a negligible effect. Finally, the temporal variation associ-

\footnotetext{
${ }^{4}$ Relativistic protons which does not produce a hadronic shower in the calorimenter and release energy only along their track.
} 


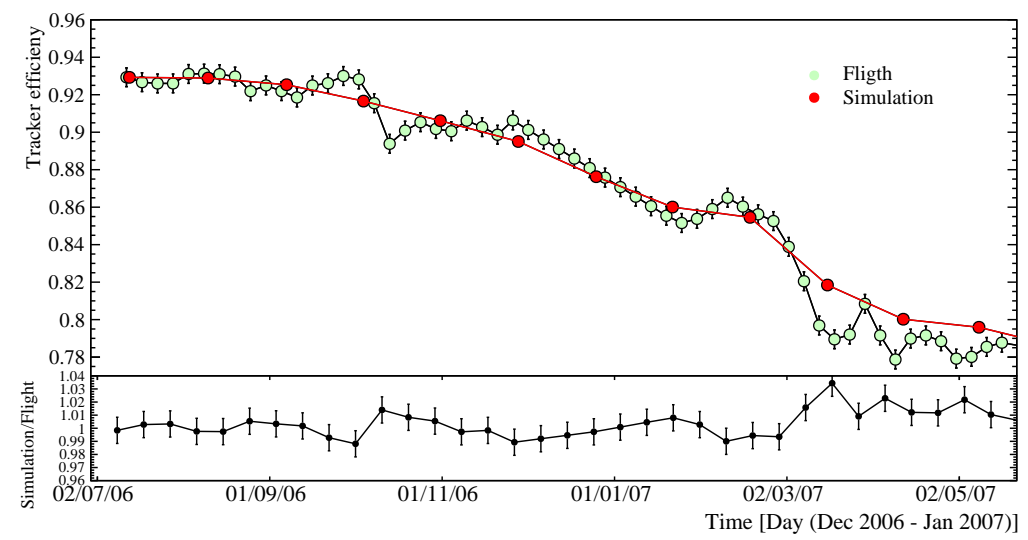

Figure 3: Top panel: the efficiency of the tracker selection as a function of time. The light green dots represent the weekly efficiency evaluated with flight data while the red dots represents the simulated efficiency averaged on one month. Bottom panel: the ratio between the simulated and flight efficiencies. The ratio was calculated interpolating the simulated efficiencies for the temporal division of the simulated efficiency.

ated with the CIRs was studied normalizing each daily fluxes on the respectively monthly average flux ${ }^{5}$.

\section{Results}

The time variation of the proton, helium and electron intensity was studied from November 2006 to March 2007 in four different rigidity interval between 0.4 and $20 \mathrm{GV}$. Additionally, thanks to the high statistics, the proton Forbush decrease was studied with a better energy resolution (twelve rigidity bins between 0.4 and $20 \mathrm{GV}$ ). The amplitude and the recovery time was studied by fitting the intensity profile with the following function:

$$
1-A e^{-\frac{t-t_{0}}{\tau}}
$$

The free parameter are the amplitude $A$, the absolute reference time $t_{0}$ and the recovery time $\tau$. With this procedure the energy dependence of the amplitude and the recovery time was studied over the twelve rigidity bin for the proton fluxes. For comparison also helium and electron behaviors were studied for the December 2006 Forbush decrease. The helium and the proton intensity show a good agreement for both the amplitude and the recovery time. On the contrary electrons shows on average a faster recovery time with respect to the protons and helium with a similar amplitude. This effect could be interpreted as a charge sign dependence introduced by drift motions which affects the GCRs during their propagation through the heliosphere [12]. During $A<0$ polarity cycles such as solar cycle 23, when the heliospheric magnetic field is directed toward the Sun in the northern hemisphere, negatively charge particles undergo drift motion mainly from the polar to the equatorial regions and outwards along the heliospheric current sheet. Positively charged particles drift mainly in opposite directions. For this reason, as the CME expands through the heliosphere

\footnotetext{
${ }^{5}$ Each day of February was normalize to the mean GCR February flux and so on.
} 
${ }^{6}$ protons are proportionally more affected from this interplanetary disturbance with respect to the electrons. This induce a longer suppression for protons with respect to electrons. Moreover, an opposite behavior during $A>0$ polarity cycle is expected since inverting the polarity of the HMF correspond to reverse the drift motion pattern between positively and negatively charged particles. In this case electron would have a slower recovery time with respect to protons.

In addition to the temporal variation produced by the CME propagation in heliosphere, a periodicity of about 13 days was observed in the proton and helium flux after the Forbush decrease. This periodicity lasted for about two months and had a mean amplitude of about $7 \%$. This temporal variations were compared with the the temporal evolution of the SW parameter like the SW velocity, the proton density, the HMF intensity and others. A correlation was found between the GCR temporal variation and some specific SW feature who could point to the passage of prominent structures of compressed plasma in the heliosphere like CIR. However, since the HCS is often crossed in the slow solar wind preceding a CIR, and may be incorporated into the CIR along with the slow solar wind, a correlation between this periodic intensity variation and the HCS crossing cannot be excluded.

\section{Conclusion}

For the first time a Forbush decrease (December 2006) was extensively studied with GCR detected directly in space with the PAMELA instrument. The amplitude and the recovery time were investigated as a function of energy. Protons, helium and electrons were measured during this event. The proton and the helium amplitude and recovery time were found in good agreement while electrons on average shows a faster recovery. This could be interpreted as a charge-sign dependence due to the different global drift pattern between proton and electrons. A 13.5 days periodicity was also measured between February and March 2006. This phenomena could be interpreted as an effect of prominent structures of compressed plasma in the solar wind (CIRs) or to the latitudinal gradient due to the crossing of the HCS.

We acknowledge partial financial support from The Italian Space Agency (ASI) under the program "Programma PAMELA - attivita' scientifica di analisi dati in fase E". We also acknowledge support from Deutsches fur Luft- und Raumfahrt (DLR), The Swedish National Space Board, The Swedish Research Council, The Russian Space Agency (Roscosmos) and Russian Science Foundation and NASA Supporting Research Grant 13 - SRHSPH1320075. M. Potgieter and J. L. Raath acknowledge the partial financial support from the South African Research Foundation (NRF) under the SA-Italy Bilateral Programme.

\section{References}

[1] E. N. Parker 1963 Interplanetary Dynamical Processes, Interscience Publishers

[2] M. S. Potgieter 2013 Living Reviews in Solar Physics 103

[3] H. V. Cane 2000 Space Science Reviews 9355

\footnotetext{
${ }^{6}$ The extend of the CME near Earth was estimated to be 74 in latitude and 117 in longitude [13] with respect to the heliospheric equator.
} 
[4] C. W. Barnes, and J. A. Simpson 1976, Astrophys. J., 20791

[5] I. G. Richardson 2004 Space Science Reviews 111267

[6] J. Simpson et al. 1992 Astron. Astrophys. Suppl. 92

[7] J. Gieseler et al. 2009 Proc. of the $31^{\text {th }}$ ICRC

[8] M. S. Potgieter et al. 2014 Sol Phys 289

[9] O. Adriani and others 2011 Astrophys. J. 742102

[10] Lucas R. Vieira and others 2012 Advances in Space Research 491615

[11] P. Picozza and others 2007 Astropart. Phys. 27296

[12] S. E. S. Ferreira and M. S. Potgieter 2004 Astropart. Phys. 603744

[13] Y. Liu and J. G. Luhmann and R. MÃijller-Mellin and P. C. Schroeder and L. Wang and R. P. Lin and S. D. Bale and Y. Li and M. H. AcuÃśa and J.-A. Sauvaudr 2008 The Astrophysical Journal 689563 